\title{
A New Efficient Permanent-Magnet Vernier Machine for Wind Power Generation
}

\author{
Jiangui $\mathrm{Li}^{1}$, K. T. Chau ${ }^{1}$, J. Z. Jiang ${ }^{2}$, Chunhua Liu ${ }^{1}$, and Wenlong $\mathrm{Li}^{1}$ \\ ${ }^{1}$ Department of Electrical and Electronic Engineering, The University of Hong Kong, Hong Kong \\ ${ }^{2}$ Department of Automation, Shanghai University, Shanghai, China
}

\begin{abstract}
This paper proposes a new outer-rotor permanent-magnet (PM) vernier machine for direct-drive wind power generation, which can offer low-speed operation to directly capture wind power, and enable high-speed rotating field design to maximize the power density. Compared with its mechanical gear counterpart, the proposed machine can eliminate the mechanical wear and tear as well as gear transmission loss, thus improving the generation reliability and efficiency. The key is to newly introduce the flux-modulation poles which can effectively modulate the high-speed rotating field of the armature windings and the low-speed rotating field of the PM outer rotor. By using the time-stepping finite-element method, the proposed machine can be accurately analyzed. Hence, its performances are quantitatively compared with other PM vernier machines, thus verifying its validity.
\end{abstract}

Index Terms-Generator, permanent-magnet (PM) machine, renewable energy, vernier machine, wind power.

\section{INTRODUCTION}

W IND power as an abundant, clean, renewable energy resource has been attracting increasing attention for solving problems arising from energy crisis and environmental pollution. Basically, wind power generation can be classified as constant-speed constant-frequency (CSCF) and variable-speed constant-frequency (VSCF) systems. The CSCF system simply adopts a constant-speed generator to supply the power grid without using any other power converter. Since the turbine speed is kept constant regardless of the variation of wind speeds, it suffers from very low efficiency and high mechanical stress. With the use of power electronics, the VSCF system can produce constant-frequency output under variable turbine speeds. Since the turbine speed changes along with the wind speed to capture the maximum wind power, its efficiency is much higher than that of the CSCF one. However, the generator of the VSCF system still suffers from the well-known matching problem: the natural wind speed $(5-10 \mathrm{~m} / \mathrm{s})$ and hence the turbine speed (100-200 rpm) are low, which cannot match with the modern high-speed electric machines. Currently, this problem is handled by either adopting a speed-boost mechanical gear [1], or using low-speed machine design [2]. The former one causes mechanical wear and tear, audible noise and low efficiency, whereas the latter one increases the generator size and weight, as well as raw material cost.

Recently, this matching problem has been solved by integrating a coaxial magnetic gear into a permanent-magnet (PM) generator [3], which allows for directly mounting wind blades onto the low-speed outer gear, and enables the generator coupled with the high-speed inner gear to operate at high speeds. However, this magnetic-geared wind generator desires a complex structure, involving two rotating bodies and three airgaps, which increases manufacture difficulty and cost.

Manuscript received October 29, 2009; accepted February 23, 2010. Current version published May 19, 2010. Corresponding author: J. Li (e-mail: jgli@eee. hku.hk).

Color versions of one or more of the figures in this paper are available online at http://ieeexplore.ieee.org.

Digital Object Identifier 10.1109/TMAG.2010.2044636
It is well known that the vernier reluctance machine can offer low-speed high-torque operation, while avoiding the increase of the number of armature winding pole-pairs [4]. In order to improve the power density, the vernier reluctance machine incorporates PMs to provide the excitation, thus becoming the PM vernier (PMV) machine, which includes the single-excitation PMV (SE-PMV) type [5] and the dual-excitation (DE-PMV) type [6]. Nevertheless, both of them are not suitable to serve as direct-drive wind generators.

The purpose of this paper is to propose a new outer-rotor PMV (OR-PMV) machine for wind power generation, which incorporates the attractive features of outer-rotor PM generator and vernier machine. In Section II, the configuration of the proposed wind power generation system will be introduced. Section III will be devoted to the design of the proposed OR-PMV machine as compared with its counterparts, namel,y the SE-PMV and DE-PMV machines. In Section IV, the mathematical model and finite element analysis of the proposed machine will be presented. In Section V, the machine performances will be assessed, and hence quantitatively compared with its counterparts. Finally, conclusion will be drawn in Section VI.

\section{SYSTEM CONFIGURATIONS}

The configuration of the conventional wind power generation system is shown in Fig. 1(a), which consists of wind blades to capture wind energy, a gearbox to match the low-speed operation of wind blades and the high-speed operation of the generator, a conventional generator to produce three-phase variable-frequency output, a bridge rectifier to perform AC-DC conversion, a DC-DC converter to regulate the DC voltage, a battery pack to store the energy, and an inverter to perform DC-AC conversion for the power grid. The major drawback of this system is the use of gearbox which is not only bulky and costly, but also suffers from transmission loss and mechanical wear and tear.

In contrast, the configuration of the proposed system is shown in Fig. 1(b) in which the wind blades are directly mounted on the outer rotor of the PMV machine while the generated output voltage is similar to that of the conventional generator. It should be noted that this configuration is very similar to that using the low-speed outer-rotor generator [2]. The difference is the need 


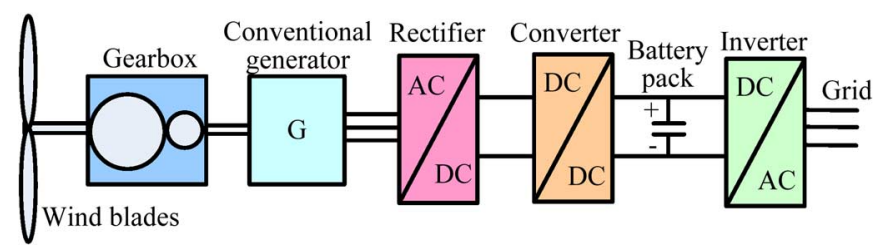

(a)

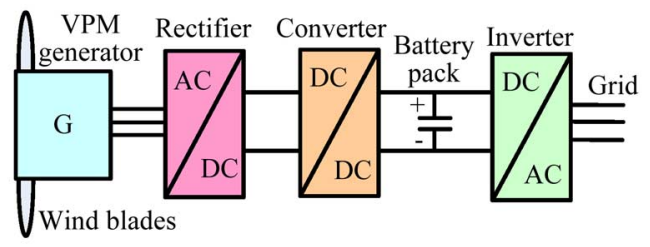

(b)

Fig. 1. Wind power generation systems. (a) Conventional. (b) Proposed.
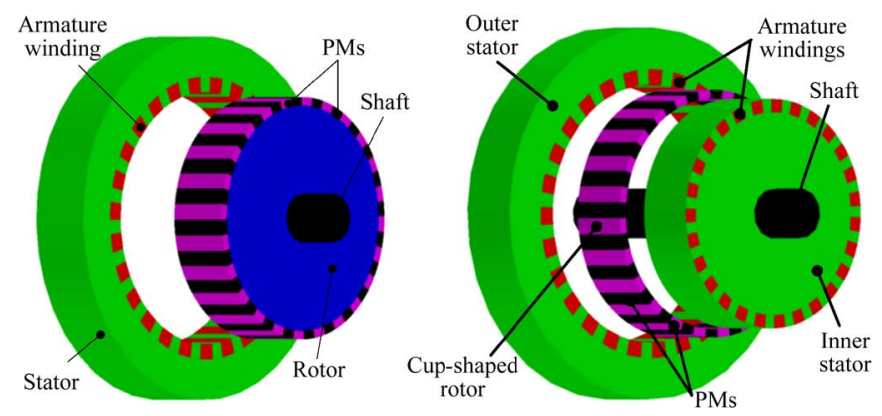

Fig. 2. Existing PMV machines. (a) SE-PMV. (b) DE-PMV.

of much larger size and heavier weight for the generator with low-speed design.

\section{Machine Design}

Fig. 2 depicts two existing types of PMV machines. The SE-PMV machine takes the merit of simplicity by using only one airgap. However, because of the number of armature winding poles is small and the number of PM poles is large, it needs to adopt open-slot drum windings in the stator and a large rotor diameter. Thus, it desires special winding fabrication and creates a large vacancy inside the rotor, leading to deteriorate its power density. Consequently, the DE-PMV machine adopts an additional stator to utilize the inner space inside the rotor, hence improving the power density. However, this machine structure involves two airgaps and a cup-shaped rotor, which is too complicated for direct-drive wind power generation.

By borrowing the concept of magnetic gears [3] and the outer-rotor arrangement of other machines [7], the proposed OR-PMV machine is shown in Fig. 3. The key is to newly introduce the flux-modulation poles (FMPs) in the outer part of the inner stator, which resemble the ferromagnetic segments of the stationary ring in the coaxial magnetic gear, to modulate the high-speed rotating field of the armature windings and the low-speed rotating field of the PM outer rotor. The corresponding armature winding connection is depicted in Fig. 4. Compared with the previous designs, the proposed machine takes the following advantages:

- This structure involves only one airgap, which is much simpler than the magnetic-geared machine structure (involving

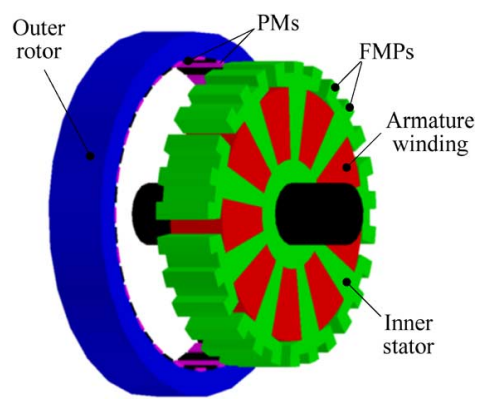

Fig. 3. Proposed OR-PMV machine.

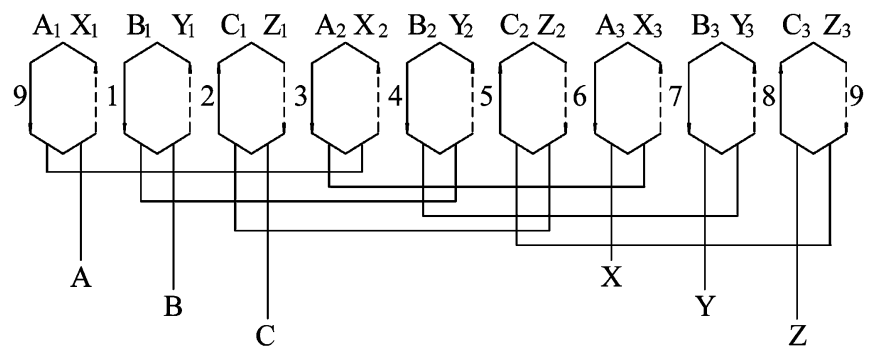

Fig. 4. Armature winding connection.

three airgaps) [3], and the DE-PMV machine (involving two airgaps) [6].

- The outer-rotor arrangement inherently provides a large diameter to accommodate a large number of PM poles, and enable full utilization of the inner space for the stator to accommodate the armature windings, thus eliminating the problem of large vacancy in the SE-PMV machine [5].

- The outer rotor allows for direct coupling with the wind blades, thus alleviating the bearing requirements and improving the mechanical integrity.

- The inner-stator arrangement with FMPs enables to adopt compact armature windings, thus avoiding the problem of drum windings used in the SE-PMV machine [5]. Also, the armature windings adopt the coil pitch equal to the slot pitch, which can minimize the end-windings, hence saving the copper material and reducing the copper loss.

Similar to the magnetic gear [3] or other PMV machines [5], [6], the proposed machine is governed by

$$
p_{r}=N_{s}-p_{s}
$$

where $N_{s}$ is the number of FMPs, $p_{r}$ is the number of rotor PM pole-pairs, and $p_{s}$ is the number of armature winding pole-pairs. Consequently, the high-to-low speed ratio $G_{r}$ is given by

$$
G_{r}=\frac{\left|m p_{s}+k N_{s}\right|}{m p_{s}}
$$

where $m=1,3, \cdots$ and $k=0, \pm 1, \pm 2, \cdots$.

In this design, the combination of $m=1$ and $k=-1$ is selected since it yields the highest asynchronous space harmonic. There are nine slots in the inner stator, which are occupied by three-phase armature windings with six poles $\left(p_{s}=3\right)$. Each stator tooth is split into three FMPs, thus constituting totally 27 FMPs $\left(N_{s}=27\right)$. From (1), $p_{r}=24$ is resulted, which denotes that there are $48 \mathrm{PM}$ poles mounting on the outer rotor. From (2), it yields $G_{r}=-8: 1$, namely the rotor speed is only $1 / 8$ 
of that in the conventional machine with the same number of armature winding pole-pairs, but rotating in an opposite direction. Therefore, when the outer rotor speed is $150 \mathrm{rpm}$, the speed of rotating field in the stator is scaled up to $1200 \mathrm{rpm}$, hence the generated output voltage is the line frequency of $60 \mathrm{~Hz}$.

\section{The TIME-STEPPING FEM ANALYSIS}

The time-stepping FEM (TS-FEM) is employed to analyze the system performances. First, the electromagnetic field equation of the machine is governed by

$$
\left\{\begin{array}{l}
\boldsymbol{\Omega}: \frac{\partial}{\partial x}\left(v \frac{\partial A}{\partial x}\right)+\frac{\partial}{\partial x}\left(v \frac{\partial A}{\partial y}\right)=-J-v\left(\frac{\partial B_{r x}}{\partial x}-\frac{\partial B_{r x}}{\partial x}\right)+\sigma \frac{\partial A}{\partial t} \\
\mathrm{~S}_{1}: A=0
\end{array}\right.
$$

where $\Omega$ is the region of calculation, $A$ is the magnetic vector potential component along the $z$ axis, $J$ is the current density, $\nu$ is the reluctivity, $\sigma$ is the electrical conductivity, $B_{r x}$, and $B_{r y}$ are, respectively, the remnant flux density components of the PM along the $x$ and $y$ axes, and $\mathrm{S}_{1}$ is the boundary of the region of calculation. Second, the armature circuit equation of the machine is expressed as

$$
\left(R+R_{l}\right) i+\left(L_{e}+L_{l}\right) \frac{d i}{d t}=\frac{l}{S} \iint_{\Omega} \frac{\partial A}{\partial t} d \Omega
$$

where $i$ is the stator phase current, $R$ and $L_{e}$ are the stator winding resistance and inductance, respectively, $R_{l}$ and $L_{l}$ are the load resistance and inductance, respectively, $S$ is the sectional area of the conductor of each phase, $l$ is the axial length of iron core. Third, the motion equation is given by

$$
J_{m} \frac{d \omega}{d t}=T_{m}-T_{e}-B \omega
$$

where $J_{m}$ is the moment of inertia, $\omega$ is the rotor speed, $T_{m}$ is the mechanical driving torque, $T_{e}$ is the electromagnetic torque, and $B$ is the damping coefficient.

After coupling (3)to (5) and applying discretization, the TS-FEM can be performed to calculate both the steady-state and dynamic performances of the machine.

\section{Performances of the Proposed Machine}

In order to illustrate the validity of the proposed machine, its performances are quantitatively compared with that of the SE-PMV and DE-PMV machines. For fair comparison, their overall outside diameters and axial lengths are the same, while their total copper volume and PM volume are similar. Their rated frequencies are designed to be all equal to the line frequency of $60 \mathrm{~Hz}$. A comparison of their key design data is summarized in Table I. It can be found that the proposed machine possesses the largest rated power and highest rated voltage for the same machine size and operating frequency. This merit is actually due to the proposed PMV structure which can significantly improve the power density. Also, the required raw materials, especially the PM volume, of the proposed machine are less than the others, leading to enhance its cost competitiveness. It should be noted that the DE-PMV machine desires much more PM material, because it needs to mount two layers of PM poles to interact with both the outer and inner stator armature windings.
TABLE I

DESIGN DATA COMPARISON

\begin{tabular}{|c|c|c|c|}
\hline & Proposed & SE-PMV & DE-PMV \\
\hline Rated power & $2.2 \mathrm{~kW}$ & $1.0 \mathrm{~kW}$ & $1.2 \mathrm{~kW}$ \\
\hline Rated phase voltage & $62 \mathrm{~V}$ & $31 \mathrm{~V}$ & $38 \mathrm{~V}$ \\
\hline Rated frequency & $60 \mathrm{~Hz}$ & $60 \mathrm{~Hz}$ & $60 \mathrm{~Hz}$ \\
\hline No. of stator pole-pairs & 3 & 3 & 3 \\
\hline No. of stator slots & 27 & 27 & $27 \times 2$ \\
\hline No. of rotor pole-pairs & 48 & 48 & 48 \\
\hline Overall outside diameter & $233 \mathrm{~mm}$ & $233 \mathrm{~mm}$ & $233 \mathrm{~mm}$ \\
\hline Shaft diameter & $40 \mathrm{~mm}$ & $40 \mathrm{~mm}$ & $40 \mathrm{~mm}$ \\
\hline Axial length & $60 \mathrm{~mm}$ & $60 \mathrm{~mm}$ & $60 \mathrm{~mm}$ \\
\hline Airgap length & $0.6 \mathrm{~mm}$ & $0.6 \mathrm{~mm}$ & $0.6 \times 2 \mathrm{~mm}^{3}$ \\
\hline Total copper volume & $507 \mathrm{~cm}^{3}$ & $500 \mathrm{~cm}^{3}$ & $513 \mathrm{~cm}^{3}$ \\
\hline Total iron volume & $2070 \mathrm{~cm}^{3}$ & $2209 \mathrm{~cm}^{3}$ & $2646 \mathrm{~cm}^{3}$ \\
\hline Total PM volume & $433 \mathrm{~cm}^{3}$ & $442 \mathrm{~cm}^{3}$ & $432 \mathrm{~cm}^{3}$ \\
\hline
\end{tabular}

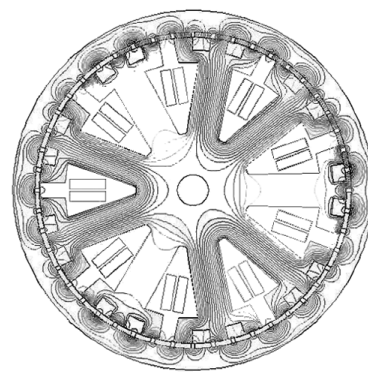

(a)

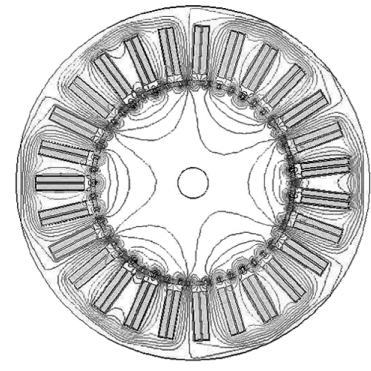

(b)

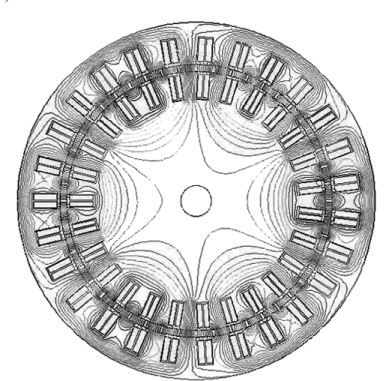

(c)
Fig. 5. No-load magnetic field distributions. (a) Proposed. (b) SE-PMV. (c) DE-PMV.

Fig. 5 shows the magnetic field distributions of the proposed machine as well as the SE-PMV and DE-PMV machines under no-load. It can be observed that the flux lines per stator tooth of the proposed machine can pass through the FMPs separately, hence verifying the desired flux modulation. The corresponding airgap flux density waveforms are shown in Fig. 6. It can be seen that all of them have 24 pole-pairs in the airgap within $360^{\circ}$ which corresponds to three pole-pairs of the stator rotating field, thus well agreeing with the principle of vernier machines. Quantitatively, the averaged peak values of the proposed machine, SE-PMV machine and DE-PMV machine are $0.944 \mathrm{~T}, 0.879 \mathrm{~T}$, and $0.8865 \mathrm{~T}, 0.8866 \mathrm{~T}$, respectively. It can be seen that the proposed machine has the highest airgap flux density, which is due to the fact that the SE-PMV machine has a larger flux leakage, and the DE-PMV has a longer effective airgap. 


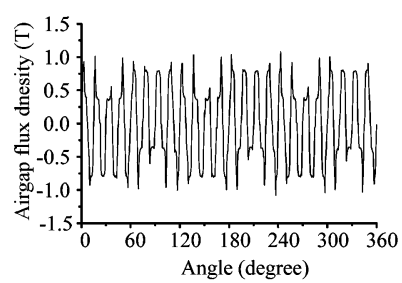

(a)

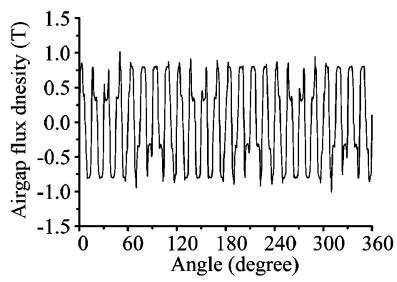

(c) (b)

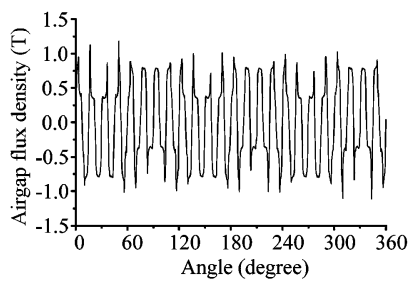

(d)

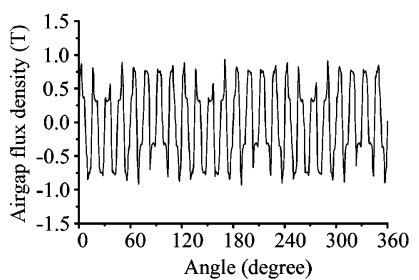

Fig. 6. No-load airgap flux density waveforms. (a) Proposed. (b) SE-PMV. (c) DE-PMV outer airgap. (d) DE-PMV inner airgap.

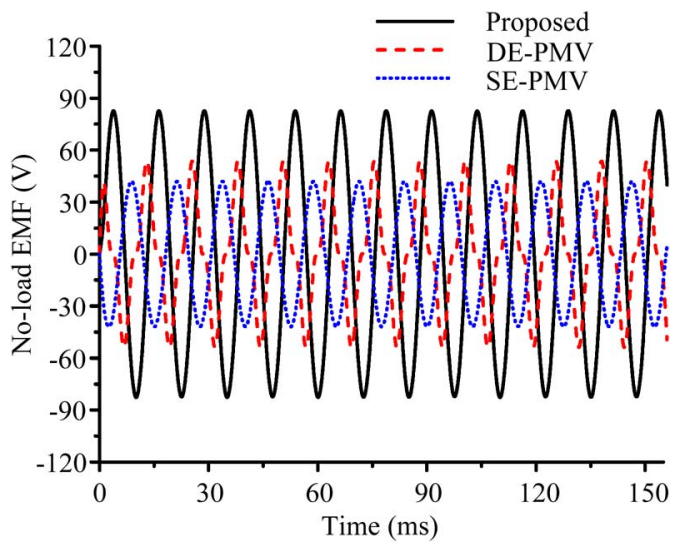

Fig. 7. No-load EMF waveforms.

Fig. 7 shows the no-load EMF waveforms of all three PMV machines at the rated speed of $150 \mathrm{rpm}$. It can be found that the root mean square (RMS) values of the proposed machine, SE-PMV machine and DE-PMV machine are 62.4 30.8, and $37.5 \mathrm{~V}$, respectively. Namely, the generated voltage of the proposed machine is $102.6 \%$ and $66.4 \%$ higher than the SE-PMV and DE-PMV counterparts, respectively. Moreover, it can be observed that the no-load EMF waveforms of the proposed machine and the SE-PMV machine are very sinusoidal, whereas the waveform of the DE-PMV machine is nonsinusoidal which is actually the characteristic due to the doubly salient structure [7].

Fig. 8 shows the electromagnetic torque waveforms of all three PMV machines at the rated condition. It can be found that the averaged steady-state torque of the proposed machine is 56.7 $\mathrm{Nm}$, while the SE-PMV machine is $33.5 \mathrm{Nm}$ and the DE-PMV machine is $37.6 \mathrm{Nm}$. Namely, the torque-handling capability of the proposed machine is $69.3 \%$ and $50.8 \%$ higher than the SE-PMV and DE-PMV counterparts, respectively. On the other hand, it can be observed that the torque ripples of the proposed machine, SE-PMV machine and DE-VPV machine are 5.9\%, $4.6 \%$, and $5.2 \%$, respectively. The torque ripple of these machines are mainly due to the cogging torque occurs at the different positions of the stator teeth with different airgap lengths. It should be noted that all these torque ripples are very accept-

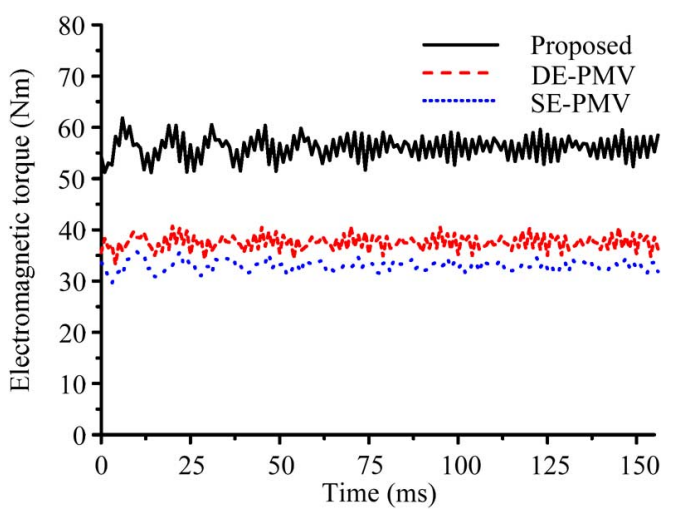

Fig. 8. Electromagnetic torque waveforms.

able, which is actually much lower than that of other doubly salient PM machines [8].

\section{CONCLUSION}

In this paper, a novel OR-PMV machine has been developed for direct-drive wind power generation, which can offer lowspeed operation to directly capture wind power, and enable highspeed rotating field design to maximize the power density. Compared with its mechanical gear counterpart, the proposed machine can eliminate the mechanical wear and tear as well as transmission loss, thus improving the generation reliability and efficiency. Compared with its PMV counterparts, it offers higher power density and higher output voltage, while reducing the raw material volume and hence the overall cost. By using the TS-FEM, the proposed machine is quantitatively compared with other PMV machines in terms of EMF generation and torquehandling capability, thus verifying that it is very promising for wind power generation.

\section{ACKNOWLEDGMENT}

This work was supported by a grant (Project No. HKU7105/ 07E) from the Hong Kong Research Grants Council, Hong Kong Special Administrative Region, China.

\section{REFERENCES}

[1] R. Datta and V. T. Ranganathan, "Variable-speed wind power generation using a doubly fed wound rotor induction machine: A comparison with alternative schemes," IEEE Power Eng. Rev., vol. 22, no. 7, pp. 52-52, 2002.

[2] J. Chen, C. V. Nayar, and L. Xu, "Design and finite-element analysis of an outer-rotor permanent magnet generator for directly coupled wind turbines," IEEE Trans. Magn., vol. 36, no. 5, pp. 3802-3809, 2000.

[3] L. Jian and K. T. Chau, "A magnetic-geared outer-rotor permanentmagnet brushless machine for wind power generation," IEEE Trans. Ind. Appl., vol. 45, no. 3, pp. 954-962, 2009.

[4] S. Taïbi, A. Tounzi, and F. Piriou, "Study of a stator current excited vernier reluctance machine," IEEE Trans. Energy Convers., vol. 21, no. 4, pp. 823-831, 2006.

[5] A. Toba and T. A. Lipo, "Generic torque-maximizing design methodology of surface permanent-magnet vernier machine," IEEE Trans. Ind. Appl., vol. 36, no. 6, pp. 1539-1546, 2000.

[6] A. Toba and T. A. Lipo, "Novel dual-excitation permanent magnet vernier machine," in Proc. Conf. Rec. IEEE-IAS Annu. Meeting, 1999, pp. 2539-2544.

[7] C. Liu, K. T. Chau, J. Z. Jiang, and L. Jian, "Design of a new outer-rotor permanent magnet hybrid machine for wind power generation," IEEE Trans. Magn., vol. 44, no. 6, pp. 1494-1497, 2008.

[8] Y. Gong, K. T. Chau, J. Z. Jiang, C. Yu, and W. Li, "Design of doubly salient permanent magnet motors with minimum torque ripple," IEEE Trans. Magn., vol. 45, no. 10, pp. 4704-4707, 2009. 